\title{
Whistler wave excitation by relativistic electrons in coronal loops during solar flares
}

\author{
C. Vocks and G. Mann
}

\author{
Astrophysikalisches Institut Potsdam, An der Sternwarte 16, 14482 Potsdam, Germany \\ e-mail: cvocks@aip.de
}

Received 12 August 2005 / Accepted 19 January 2006

\begin{abstract}
Whistler wave packets propagating along magnetic loops are the standard model for the generation of the fiber bursts that are observed in dynamical radio spectra during solar flares. The whistlers are believed to be generated by electron-loss cone distributions that form in the loop, if energetic electrons that have been injected by the flare are mirrored at the loop footpoints. A relativistic method for calculating plasma wave growth/damping rates for given particle velocity distribution functions (VDFs) is used to study the wave generation in dependence on the wave frequency and on the wave propagation angle with respect to the background magnetic field. Due to a Taylor expansion of the plasma dispersion function, it yields better accuracy for small growth rates than does a direct solution of the dispersion relation for a complex frequency. The results show that the whistler wave generation is limited to small propagation angles with respect to the background magnetic field. The wave growth rates are found to be high enough to account for the properties of fiber bursts observed in solar radio data.
\end{abstract}

Key words. radiation mechanisms: non-thermal - plasmas - waves - Sun: corona - Sun: radio radiation - Sun: flares

\section{Introduction}

Solar flares accelerate electrons to high energies (Lin 1974) and lead to the generation of various types of radio emission, see e.g. Warmuth \& Mann (2004) as a review. Figure 1 shows a hard X-ray photon spectrum of the flare of 28 October 2003 as observed by the RHESSI satellite (Lin et al. 2002). The photon spectrum clearly shows the presence of a nonthermal power-law component and additionally thermal emission. Supposing thick target bremsstrahlung (Brown 1971), an injected electron-flux spectrum with a power-law index of $\delta=3.8$ can be derived from the photon spectrum (for the method, see e.g. Holman et al. 2003). Such values are typical of solar flares.

These highly energetic electrons can be trapped in coronal loops. They are mirrored at the loop footpoints and can establish a loss-cone distribution (Croley et al. 1978). Such a distribution is unstable and gives rise to whistler wave excitation (Scharer \& Trivelpiece 1967; Mann et al. 1989). If these whistlers nonlinearly coalescence with high-frequency plasma waves, e.g. Langmuir waves, into radio waves, they can be observed as fiber (or intermediary drift) bursts (Kuijpers 1975; Mann et al. 1987; Benz \& Mann 1998; Aurass et al. 2005) in the solar radio radiation, e.g. within type IV radio bursts.

Figure 2 shows fiber bursts in the dynamical radio spectrum that was recorded by the Observatory of Solar Radioastronomy of the Astrophysical Institute Potsdam (Mann et al. 1992) during the event of 28 October 2003 at the same time as the photon spectrum from Fig. 1 was taken. Fiber bursts appear as stripes of enhanced radio emission in the dm-range $(\approx 400-800 \mathrm{MHz})$ in dynamical radio spectra. They are drifting from high to low frequencies with (intermediate) drift rates between those of type II and type III radio bursts (Kuijpers 1975).

In previous studies, e.g. (Scharer \& Trivelpiece 1967; Mann et al. 1989), the energetic electrons are considered to be nonrelativistic. The recent paper by Aurass et al. (2005) shows that

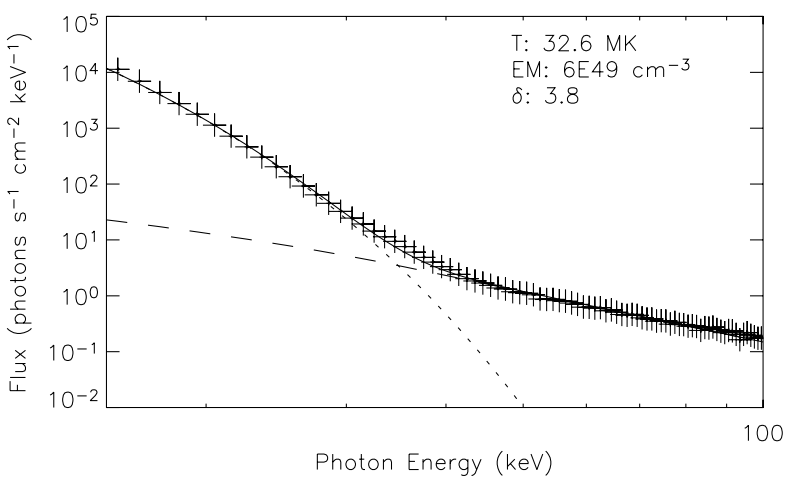

Fig. 1. Hard X-ray photon spectrum of the flare on 28 October 2003, 11:17:00-11:17:20 UT, as observed by the RHESSI satellite. The spectrum shows a thermal (dotted line) and nonthermal power-law component (dashed line), as well as the power-law index $\delta=3.8$ of the derived electron-flux spectrum.

the whistler waves associated with fiber bursts are generated by relativistic electrons. The study presented in this paper is motivated by this result, which requires treating the whistler wave excitation within a relativistic framework.

In order to investigate whistler-wave generation, it is necessary to have a method of calculating plasma wave growth/damping rates for a given electron velocity distribution function (VDF), e.g. a loss cone VDF. Since electron energies of several tens of $\mathrm{keV}$ are to be considered during solar flares, a relativistic description of the electrons is necessary, see e.g. Melrose (1980).

The wave growth/damping rate, $\gamma$, is described as the imaginary part of the complex frequency

$\omega=\omega_{r}+\mathrm{i} \gamma$ 


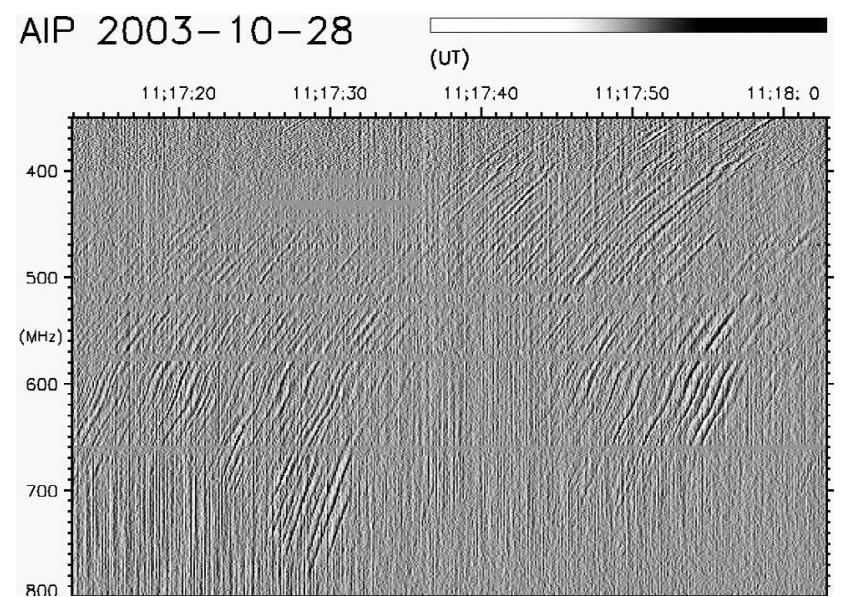

Fig. 2. A typical part of a patch of fiber bursts in the dynamical radio spectrum of 28 October 2003. The image shows the spectrum's temporal derivative. The data were recorded by the Observatory of Solar Radioastronomy of the Astrophysical Institute Potsdam.

with $\omega_{r}$ the real part of the frequency, and $i^{2}=-1$. For a plane wave setup involving a factor $\exp (-\mathrm{i} \omega t)$, a negative $\gamma$ corresponds to wave damping and a positive one to wave growth.

Matsuda \& Smith (1992) have developed a code for calculating the complex frequency, $\omega$, that aims for a solution of the dispersion relation

$D(\omega, \boldsymbol{k})=0$

for a given wave vector, $\boldsymbol{k}$. There $D(\omega, \boldsymbol{k})$ is the plasma dispersion function. This yields both the wave frequency, $\omega_{r}$, and the growth rate, $\gamma$.

This method is based on finding a solution of the dispersion relation by varying $\omega$ for a given $\boldsymbol{k}$. Matsuda \& Smith (1992) apply their program on highly unstable plasmas with $\gamma \approx 10^{-3} \Omega_{\mathrm{e}}$, where $\Omega_{\mathrm{e}}=e B / m_{\mathrm{e}}$ is the electron cyclotron frequency in the magnetic field $B$.

In astrophysical plasmas, however, wave growth rates are often found to be much smaller, e.g. $10^{-8} \Omega_{\mathrm{e}}$ as shown in this paper. In such a case, the order-of-magnitude difference between $\gamma$ and a wave frequency near the electron cyclotron frequency is dangerously close to the limited numerical accuracy of a computer, that is typically $10^{-8}$. For accurate determination of $\gamma$ in such a plasma, a different approach is necessary.

In many textbooks on plasma physics, e.g. (Baumjohann \& Treumann 1996), a Taylor expansion around a solution of the dispersion relation $D(\omega, \boldsymbol{k})=0$ is presented:

$\gamma(\omega, \boldsymbol{k})=-\frac{\operatorname{Im}\left(D\left(\omega_{r}, \boldsymbol{k}\right)\right)}{\left.\partial \operatorname{Re}(D(\omega, \boldsymbol{k}) / \partial \omega)\right|_{\gamma=0}}$.

In Eq. (3) the numerical accuracy of $\gamma$ is determined by that of the calculation of the real and imaginary parts of the dispersion function, and not by the difference between $\gamma$ and $\omega_{r}$. Furthermore, Eq. (3) has the advantage that $D(\omega, \boldsymbol{k})$ is only evaluated in the special case $\omega=\omega_{r}$, i.e. $\gamma=0$. This greatly simplifies the numerical procedure for determining $D(\omega, \boldsymbol{k})$.

This paper is organized as follows. In the next section, the method of calculating wave growth rates is explained. In Sect. 3, it is applied to whistler wave generation by electron loss-cone distributions as they can be found in flaring loops in the solar corona. The paper closes with the conclusions and a summary in Sect. 4.

\section{Calculation of wave growth rates for relativistic electrons}

In order to study the generation of whistler waves in flaring loops, it is necessary to calculate the growth rate, $\gamma$, of a wave with frequency $\omega_{r}$ that propagates through a plasma with a given electron VDF. The angle $\theta$ between the wave vector, $\boldsymbol{k}$, and the background magnetic field, $\boldsymbol{B}$ is also given. Under these constraints, the dispersion function $D\left(\omega, k \boldsymbol{e}_{\theta}\right)$ is well-defined for any wavenumber $k$, where $\boldsymbol{e}_{\theta}$ is a unit vector that points in the direction indicated by $\theta$. The first step is to find the wavenumber of the wave mode under study, i.e. a zero of $D\left(\omega, k \boldsymbol{e}_{\theta}\right)$ as a function of $k$. Since we are interested in small $\gamma \ll \omega_{r}$, the unknown imaginary part of the complex frequency is neglected, and $\omega$ is replaced by $\omega_{r}$. Then, a zero of the real part of the dispersion function is sought:

$\operatorname{Re}\left(D\left(\omega_{r}, k \boldsymbol{e}_{\theta}\right)\right)=0$.

It is possible that this equation has several solutions with different wavenumbers $k$ that represent different wave modes. In that case, the wave polarizations of the solutions and the cold plasma dispersion relation can be used to identify the correct mode.

Once the wavenumber $k$ and thus the wave vector $\boldsymbol{k}$ has been determined, the real and imaginary parts of the dispersion function $D$ can be evaluated. The frequency derivative of the real part can easily be calculated by finite differences. Equation (3) then yields the wave growth rate. In the next subsections, the dispersion function $D(\omega, \boldsymbol{k})$ for a given electron VDF that covers relativistic energies is presented, and the methods of calculating the numerical values of its real and imaginary parts are shown.

\subsection{Derivation of the dispersion function $D(\omega, k)$}

The derivation of the dispersion function $D(\omega, \boldsymbol{k})$ can be found in many textbooks of plasma physics (e.g. Montgomery \& Tidman 1964; Melrose 1986). The formulae are presented here in order to provide a self-contained description of the calculations.

The dispersion function can be written as

$D(\omega, \boldsymbol{k})=\operatorname{det}\left(\frac{c^{2}}{\omega^{2}}\left(\boldsymbol{k} \boldsymbol{k}-1 k^{2}\right)+\epsilon(\omega, \boldsymbol{k})\right)$

with the dielectric tensor

$$
\begin{aligned}
\epsilon(\omega, \boldsymbol{k})= & \left(1-\frac{\omega_{\mathrm{p}}^{2}}{\omega^{2}}\right) 1 \\
& -\sum_{s} \frac{2 \pi \omega_{\mathrm{p}, s}^{2}}{N_{s} \omega^{2}} \sum_{l=-\infty}^{\infty} \int_{0}^{\infty} p_{\perp} \mathrm{d} p_{\perp} \int_{-\infty}^{\infty} \mathrm{d} p_{\|} \\
& \times\left(k_{\|} \frac{\partial f_{s}}{\partial p_{\|}}+\frac{l q_{s} B}{p_{\perp}} \frac{\partial f_{s}}{\partial p_{\perp}}\right) \frac{1}{\gamma_{\mathrm{L}}} \frac{\mathrm{S}_{l}\left(p_{\perp}, p_{\perp}\right)}{k_{\|} p_{\|}+l q_{s} B-m_{s} \gamma_{\mathrm{L}} \omega}
\end{aligned}
$$

and the matrix $\mathrm{S}_{l}$ defined as:

$\mathrm{S}_{l}\left(p_{\|}, p_{\perp}\right)=\left(\begin{array}{ccc}\frac{l^{2} q_{s}^{2} B^{2}}{k_{\perp}^{2}} J_{l}^{2} & \mathrm{i} \frac{l p_{\perp} q_{s} B}{k_{\perp}} J_{l} J_{l}^{\prime} & \frac{l p_{\|} q_{s} B}{k_{\perp}} J_{l}^{2} \\ -\mathrm{i} \frac{l p_{\perp} q_{s} B}{k_{\perp}} J_{l} J_{l}^{\prime} & p_{\perp}^{2}\left(J_{l}^{\prime}\right)^{2} & -\mathrm{i} p_{\|} p_{\perp} J_{l} J_{l}^{\prime} \\ \frac{l p_{\|} q_{s} B}{k_{\perp}} J_{l}^{2} & \mathrm{i} v p \| p_{\perp} J_{l} J_{l}^{\prime} & p_{\|}^{2} J_{l}^{2}\end{array}\right)$.

The lower indices $\|$ and $\perp$ denote vector components both parallel and perpendicular to the background magnetic field, respectively. $\gamma_{\mathrm{L}}=\left(1-v^{2} / c^{2}\right)^{-1 / 2}=\left(1+p^{2} /\left(m^{2} c^{2}\right)\right)^{1 / 2}$ is the Lorentz 
factor. The order of the resonance is denoted by $l$, and $J_{l}$ represents the Bessel function of order $l, J_{l}(x)$. It is evaluated at $x=k_{\perp} p_{\perp} /\left(q_{s} B\right)$, while $J_{l}^{\prime}$ represents its derivative in $x$. The dielectric tensor contains contributions from all particle species $s$ in the plasma, with charge $q_{s}$, rest mass $m_{s}$, and number density $N_{s}$.

The dielectric tensor with its integral over momentum space has to be evaluated for given gyrotropic particle VDFs, $f_{s}\left(p_{\|}, p_{\perp}\right)$. This integration is complicated by the resonance denominator $\left(k_{\|} p_{\|}+l q_{s} B-m_{s} \gamma_{\mathrm{L}} \omega_{r}\right)^{-1}$ that introduces a singularity for those $\left(p_{\|}, p_{\perp}\right)$ that meet the resonance condition:

$k_{\|} p_{\|}+l q_{s} B-m_{s} \gamma_{\mathrm{L}} \omega=0$.

\subsection{Splitting the dielectric tensor, $\epsilon(\omega, k)$}

The Taylor expansion of the dispersion function $D(\omega, \boldsymbol{k})$ in Eq. (3) only requires an evaluation of the dielectric tensor in the limit $\gamma \rightarrow 0$, i.e. $\omega \rightarrow \omega_{r}$. This greatly simplifies the integration over momentum space in Eq. (6). In this limit, factor $1 / \omega^{2}$ simply turns into $1 / \omega_{r}^{2}$. The resonance denominator $\left(k_{\|} p_{\|}+l q_{s} B-\right.$ $\left.m_{s} \gamma_{\mathrm{L}} \omega\right)^{-1}$ needs some more attention. According to Melrose (1980), it can be split into its real and imaginary parts:

$$
\begin{aligned}
& \frac{1}{k_{\|} p_{\|}+l q_{s} B-m_{s} \gamma_{\mathrm{L}} \omega}= \\
& \frac{k_{\|} p_{\|}+l q_{s} B-m_{s} \gamma_{\mathrm{L}} \omega_{r}}{\left(k_{\|} p_{\|}+l q_{s} B-m_{s} \gamma_{\mathrm{L}} \omega_{r}\right)^{2}+m_{s}^{2} \gamma_{\mathrm{L}}^{2} \gamma^{2}} \\
& +\mathrm{i} \frac{m_{s} \gamma_{\mathrm{L}} \gamma}{\left(k_{\|} p_{\|}+l q_{s} B-m_{s} \gamma_{\mathrm{L}} \omega_{r}\right)^{2}+m_{s}^{2} \gamma_{\mathrm{L}}^{2} \gamma^{2}} .
\end{aligned}
$$

In the limit $\gamma \rightarrow 0$, the real part simply equals $\left(k_{\|} p_{\|}+l q_{s} B-\right.$ $\left.m_{s} \gamma_{\mathrm{L}} \omega_{r}\right)^{-1}$, but the imaginary part introduces the Dirac delta function:

$$
\begin{gathered}
\lim _{\gamma \rightarrow 0} \frac{m_{s} \gamma_{\mathrm{L}} \gamma}{\left(k_{\|} p_{\|}+l q_{s} B-m_{s} \gamma_{\mathrm{L}} \omega_{r}\right)^{2}+m_{s}^{2} \gamma_{\mathrm{L}}^{2} \gamma^{2}}= \\
\pi \delta\left(k_{\|} p_{\|}+l q_{s} B-m_{s} \gamma_{\mathrm{L}} \omega_{r}\right) .
\end{gathered}
$$

Due to this split of the resonance denominator, the sums and integrals in Eq. (6) can be evaluated independently for both parts. Thus, it is possible to split the dielectric tensor into a "real" and an "imaginary" part:

$\epsilon=\operatorname{Re}(\epsilon)+\operatorname{iIm}(\epsilon)$.

The terms "real" and "imaginary" are set in parenthesis, since the matrix $S_{l}$ still contains complex elements. They can be written as:

$$
\begin{aligned}
\operatorname{Re}(\epsilon) & =\left(1-\frac{\omega_{\mathrm{p}}^{2}}{\omega_{r}^{2}}\right) 1-\sum_{s} \frac{2 \pi \omega_{p, s}^{2}}{N_{\mathrm{e}} \omega_{r}^{2}} \sum_{l=-\infty}^{\infty} \int_{0}^{\infty} p_{\perp} \mathrm{d} p_{\perp} \int_{-\infty}^{\infty} \mathrm{d} p_{\|} \\
& \times\left(k_{\|} \frac{\partial f_{s}}{\partial p_{\|}}+\frac{l q_{s} B}{p_{\perp}} \frac{\partial f_{s}}{\partial p_{\perp}}\right) \frac{1}{\gamma_{\mathrm{L}}} \frac{\mathrm{S}_{l}}{k_{\|} p_{\|}+l q_{s} B-m_{s} \gamma_{\mathrm{L}} \omega_{r}}
\end{aligned}
$$

and

$$
\begin{aligned}
& \operatorname{Im}(\epsilon)=-\sum_{s} \frac{2 \pi \omega_{p, s}^{2}}{N_{\mathrm{e}} \omega_{r}^{2}} \sum_{l=-\infty}^{\infty} \int_{0}^{\infty} p_{\perp} \mathrm{d} p_{\perp} \int_{-\infty}^{\infty} \mathrm{d} p_{\|} \\
& \quad \times\left(k_{\|} \frac{\partial f_{s}}{\partial p_{\|}}+\frac{l q_{s} B}{p_{\perp}} \frac{\partial f_{s}}{\partial p_{\perp}}\right) \frac{1}{\gamma_{\mathrm{L}}} \mathrm{S}_{l} \pi \delta\left(k_{\|} p_{\|}+l q_{s} B-m_{s} \gamma_{\mathrm{L}} \omega_{r}\right) .
\end{aligned}
$$

The "real" part is of Hermitian and the "imaginary" part is of anti-Hermitian structure. They are associated with the reactive and dissipative parts of the plasma's response to the wave field, respectively (Melrose 1986).

\subsection{Evaluation of the dielectric tensor}

The "real" part of the dielectric tensor as defined in Eq. (12) is a complicated integral over the whole momentum space that contains momentum derivatives of the particle VDF. It still has a resonance denominator that leads to a singularity if the resonance condition (8) is met. It is very complicated to evaluate this integral numerically for an arbitrary VDF.

However, it can be simplified considerably. If the VDF has no short-periodic (in momentum space) variations, the momentum derivatives can be expected to take their highest values if the $\operatorname{VDF} f_{s}\left(p_{\|}, p_{\perp}\right)$ itself has its highest values, i.e. within the thermal core of the VDF. Thus, the thermal core of the distribution dominates the "real" part of the dielectric tensor. In space plasma physics, the thermal core of a particle distribution is typically close to a Maxwellian VDF. Thus, it is reasonable to calculate $\operatorname{Re}(\epsilon)$ for a Maxwellian VDF that has the same particle number density and temperature as the $\operatorname{VDF} f_{s}\left(p_{\|}, p_{\perp}\right)$.

The fact that the thermal speed is nonrelativistic, $v_{\text {th }} \ll c$, even for electrons in the solar corona, further simplifies Eq. (12). The electron thermal speed for electrons with a temperature of, e.g., $T=2 \times 10^{6} \mathrm{~K}$ is $v_{\text {th }}=5500 \mathrm{~km} \mathrm{~s}^{-1}$. Thus, the thermal core and its contribution to $\operatorname{Re}(\epsilon)$ can be described in the nonrelativistic limit with a Lorentz factor $\gamma_{\mathrm{L}}=1$. This simplifies Eq. (12) considerably, since it removes a complicated dependence on the momentum coordinate and enables an analytic solution of the integral for a Maxwellian VDF.

Inserting a Maxwellian VDF, $f_{s, M}\left(p_{\|}, p_{\perp}\right)$, into Eq. (12) enables further analytic treatment of $\operatorname{Re}(\epsilon)$. Despite the singularity, the integral has a well-defined value, and the resulting matrix elements are closely related to the well-known plasma dispersion function.

The evaluation of the "imaginary" part of the dielectric tensor, Eq. (13), is simplified considerably by the Dirac delta distribution. The integral over momentum space is reduced to a line integral along the solution of the resonance condition, Eq. (8). It is noteworthy that the geometry of the line $p_{\perp}\left(p_{\|}\right)$is that of a conic section (see e.g. Melrose 1986).

For a given $\left(\omega_{r}, \boldsymbol{k}\right)$, only the part of the VDF along the line $p_{\perp}\left(p_{\|}\right)$contributes to $\operatorname{Im}(\epsilon)$. This method has the advantage that the integration path might avoid the thermal core of the VDF, and cross a structure of the distribution that provides free energy for wave growth, e.g. a loss cone. In that case, the integral is not affected by the much higher phase-space density of the thermal core, and numerical errors due to the orders-ofmagnitude difference between the phase-space densities cannot occur. This is why the method used in this paper enables an accurate treatment of small wave growth/damping rates.

\subsection{Calculation of the dispersion function}

With the numerical values for the dielectric tensor at hand, the dispersion function, Eq. (5), can now be calculated easily for a given $\left(\omega_{r}, \boldsymbol{k}\right)$ and particle VDFs $f_{s}\left(p_{\|}, p_{\perp}\right)$. Inserting the results into Eq. (3) finally yields the wave growth rate.

It turns out that the imaginary part of the dispersion function, $D_{i}$, is of first order in the "imaginary" part of the dielectric tensor. Thus, if the resonance line through momentum space only traverses regions with low values of the VDF, $D_{i}$, and by means of Eq. (3) the wave growth rate, will also be low.

On the other hand, the real part of the dispersion function, $D_{r}$, is of second order in the "imaginary" part of the dielectric tensor. Since this term is small for small wave growth rates, this justifies the use of the real part of the dispersion function 
as a substitute for finding a zero of the dispersion function in Eq. (4) for calculating the wavenumber of the wave mode under consideration.

\section{Whistler wave generation in flaring loops}

With the method from the previous section, the wave growth caused by an electron loss cone distribution that forms in a magnetic loop during a solar flare can now be investigated. Loss cone VDFs are well known for emitting plasma waves. They are discussed as the sources of Jupiter's decametric radiation (Wu \& Freund 1977) and Earth's auroral kilometric radiation (Wu \& Lee 1979). Vocks \& Mann (2004) have shown that wave generation from loss cones is also possible in solar coronal funnels under quiet conditions, but the waves are heavily damped in the outer layers of the solar atmosphere. These are examples of X-mode emission that is bound to the condition that the local plasma frequency is smaller than the electron gyrofrequency, $\omega_{\mathrm{p}}<\Omega_{\mathrm{e}}$ (Wu \& Freund 1977). In a flaring loop, the electron density is far too high to meet this requirement. But whistler waves can be generated, as shown below.

\subsection{Loss cone distributions}

The electrons that are injected into the magnetic loop propagate along the magnetic field lines down to the loop footpoints. The magnetic field increases towards the footpoints, and due to conserving the magnetic moment, the electrons are mirrored close to the footpoint and reflected back into the loop.

But the smaller the initial electron pitch angle, the further an electron can go down the loop before it is mirrored. Electrons with sufficiently small pitch angles can penetrate deep into the solar atmosphere and are scattered and thermalized in the cool and dense transition region and chromosphere. Since these electrons are not reflected back into the corona, a loss-cone electron distribution forms in the flaring loop. Loss-cone distributions are well known for emitting whistler waves, as observed in the solar corona (Kuijpers 1975), Earth's (Helliwell 1975), and Jupiter's (Xiao et al. 2003) magnetosphere.

The objective of this section is to calculate whistler-wave growth rates for a loss-cone distribution that it is typical of a flaring loop, and to investigate whether the wave growth is fast enough to account for the fiber-burst observations.

The model electron VDF has two components, the thermal background plasma of the solar corona and a hot, nonthermal component that has been produced by the flare. The background plasma has a Maxwellian VDF $f_{\mathrm{b}}(p)$ with a temperature of $T_{\mathrm{b}}=1.4 \times 10^{6} \mathrm{~K}$ and a density of $N_{\mathrm{b}}=1.33 \times 10^{15} \mathrm{~m}^{-3}$, which corresponds to a plasma frequency of $f_{\mathrm{p}}=327 \mathrm{MHz}$ where fiber bursts usually are observed (Aurass et al. 2005), see Fig. 2. The magnetic field has been set to $B=3 \mathrm{G}$, in agreement with the data analysis of Aurass et al. (2005). These plasma conditions correspond to a ratio of $\omega_{\mathrm{p}} / \Omega_{\mathrm{e}}=39$ between the plasma frequency, $\omega_{\mathrm{p}}=\sqrt{N_{\mathrm{e}} e^{2} /\left(m_{\mathrm{e}} \epsilon_{0}\right)}$, and the electron cyclotron frequency, $\Omega_{\mathrm{e}}=e B / m_{\mathrm{e}}$. Such high values of $\omega_{\mathrm{p}} / \Omega_{\mathrm{e}} \gg 1$ are typical of the solar corona (Vocks \& Mann 2004). The nonthermal component of the electron VDF has a kappa distribution

$$
f_{\mathrm{h}}(p)=\frac{N_{\mathrm{h}} \Gamma(\kappa+1)}{\left(\pi(2 \kappa-3) p_{\mathrm{th}}^{2}\right)^{3 / 2} \Gamma(\kappa-1 / 2)}\left(1+\frac{p^{2}}{(2 \kappa-3) p_{\mathrm{th}}^{2}}\right)^{-(\kappa+1)}
$$

that results in a power law spectrum for high energies, where $p_{\text {th }}=\sqrt{k_{\mathrm{B}} T_{\mathrm{h}} m_{\mathrm{e}}}$ is the "thermal momentum" of the electrons,

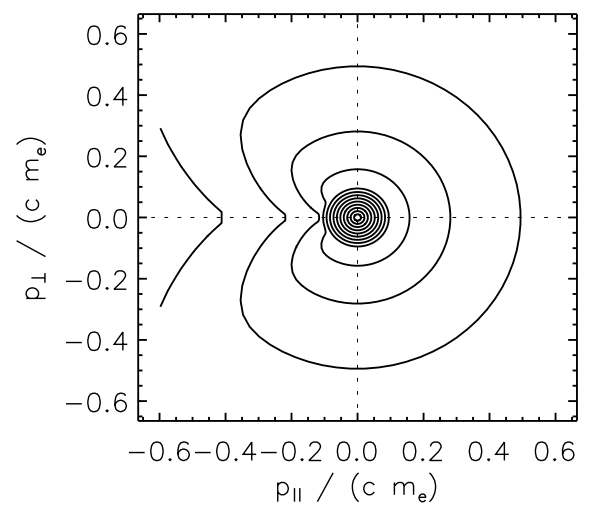

Fig. 3. Electron model VDF for a loss-cone opening angle $\theta_{\mathrm{L}}=40^{\circ}$. The isolines are chosen in such a way that they would form equidistant circles for a Maxwellian VDF.

and $\Gamma(x)$ the gamma function. For the calculations presented here, we have chosen a value $\kappa=4$. This choice provides strong suprathermal tails of the VDF and agrees well with the power law coefficient $\delta=3.8$ that can be derived (Brown 1971) from the electron-flux spectrum in Fig. 1. The numerical values for the temperature and density of the hot component of our model plasma are $T_{\mathrm{h}}=10^{7} \mathrm{~K}$ and $N_{\mathrm{h}}=1.33 \times 10^{9} \mathrm{~m}^{-3}=10^{-6} N_{\mathrm{b}}$.

Within the loss cone, the hot component vanishes. With $\theta_{\mathrm{L}}$ being the loss cone opening angle and $\Delta \theta$ the width of the transition from the loss cone to the undisturbed hot component, the total model electron VDF is defined as:

$f\left(p, \theta_{\mathrm{p}}\right)=f_{\mathrm{b}}(p)+\frac{1}{2}\left(1+\tanh \left(\frac{\left(\pi-\theta_{\mathrm{L}}\right)-\theta_{\mathrm{p}}}{\Delta \theta}\right)\right) f_{\mathrm{h}}(p)$.

The coordinate system is defined in such a way that the coordinate axis parallel to the background magnetic field points towards the loop footpoint where the mirroring and loss-cone formation takes place. This is why the loss cone is in the range of high pitch angles $\theta_{\mathrm{p}} \approx \pi$, i.e. at negative $p_{\|}$. In this paper, a losscone transition width $\Delta \theta=10^{\circ}$ has been chosen.

Figure 3 shows the model electron VDF for a loss-cone opening angle of $\theta_{\mathrm{L}}=40^{\circ}$. The thermal core and the extended hot component are both clearly visible, as is the loss cone. The model VDF covers electron energies up to $100 \mathrm{keV}$ that necessitate a relativistic method for calculating wave growth rates.

\subsection{Whistler-wave growth rates}

With this model electron VDF, it is possible to apply the method from Sect. 2 to calculate the growth rates, $\gamma$, for whistler waves as a function of wave frequency, $\omega_{r}$, and wave propagation angle, $\theta$.

Figure 4 displays the whistler-wave growth rates that result from the electron VDF in Fig. 3. An area with positive $\gamma$, i.e. whistler-wave generation, can be seen clearly. But this area is limited both in frequency and wave-propagation angle. The frequencies with wave growth have an upper limit of approximately $\omega_{r, \max }=0.07 \Omega_{\mathrm{e}}$. The limit on the wave-propagation angle, $\theta<0.15^{\circ}$, is even more restrictive. Only whistlers that propagate almost parallel to the background magnetic field are generated.

These limits on $\left(\omega_{r}, \theta\right)$ are due to the shape of the electron VDF and the resonance condition, Eq. (8). The sign of the electron charge has been considered in the definition of the dielectric tensor in Eq. (6), $q_{s}=-e$. For waves that propagate parallel 

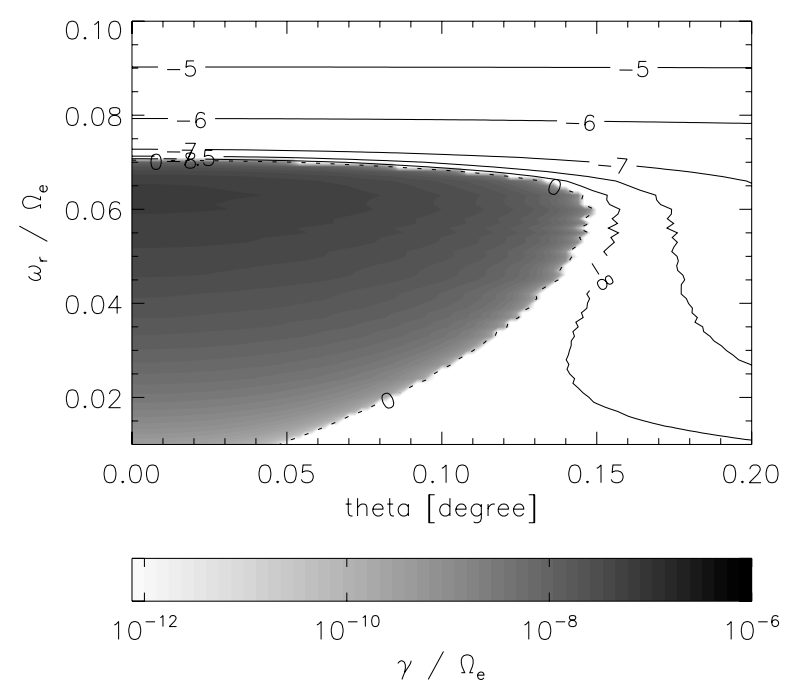

Fig. 4. Growth rates for whistler waves as a function of wave frequency, $\omega_{r}$, and propagation angle, $\theta$. Positive growth rates, $\gamma>0$, are displayed as a greyscale plot, and wave damping rates, $\gamma<0$, by isolines marked with numbers that denote an exponent, e.g. -7 corresponds to $\gamma / \Omega_{\mathrm{e}}=-10^{-7}$.

to the background magnetic field, the perpendicular wave number vanishes, $k_{\perp}=0$. For these waves, only the resonance order $l=-1$ provides a contribution to the dispersion function, Eq. (5). For $l=-1$, it follows from the resonance condition, Eq. (8), that the momentum coordinate parallel to the background magnetic field, $p_{\|}$, is negative for an electron that is in resonance with a whistler wave with frequency $\omega_{r}$. The absolute value of $p_{\|}$decreases as $\omega_{r}$ increases. At a frequency of $\omega_{r}=0.07 \Omega_{\mathrm{e}}$ the resonance momentum $p_{\|}$leaves the loss cone and enters the thermal core of the electron VDF that is clearly visible in Fig. 3. Since the electron VDF is Maxwellian there, there is no free energy available for wave growth, and the wave is strongly damped.

The resonance condition and the structure of the dielectric tensor is also the reason for the strict limitation of the wave growth to very small propagation angles $\theta<0.15^{\circ}$. For $k_{\perp}=0$, only the resonance order $l=-1$ contributes to the dispersion function, but for $k_{\perp} \neq 0$, all $l=0, \pm 1, \pm 2, \ldots$ provide a contribution. These contributions are of first or higher order in $k_{\perp}$ and thus seem to be small for small $k_{\perp}$, but nevertheless they can dominate the resulting wave growth rate. For $l=0$, the nonrelativistic resonance condition simply reads $v_{\|}=\omega_{r} / k_{\|} \approx v_{\mathrm{ph}}$. Thus, an electron that moves with the wave phase speed is in the $l=0$ resonance. For our model plasma, this speed corresponds to less than one electron thermal speed. Thus, the nonrelativistic limit may be used here, and the wave is in resonance with the thermal core of the electron VDF.

The integration over the resonance line in momentum space that leads to the "imaginary" part of the dielectric tensor, Eq. (13), now traverses the thermal core of the electron VDF for $l=0$. The phase-space density there is many orders of magnitude higher than in the loss cone where the $l=-1$ resonance has its integration path. As a result, the wave damping of the thermal core dominates the result for the wave growth rate, $\gamma$, even for very small propagation angles $\theta \approx 0.15^{\circ}$.

\subsection{Maximum wave growth rates}

Due to this influence of other resonance orders than $l=-1$ for wave propagation angles $\theta>0$, waves that propagate strictly

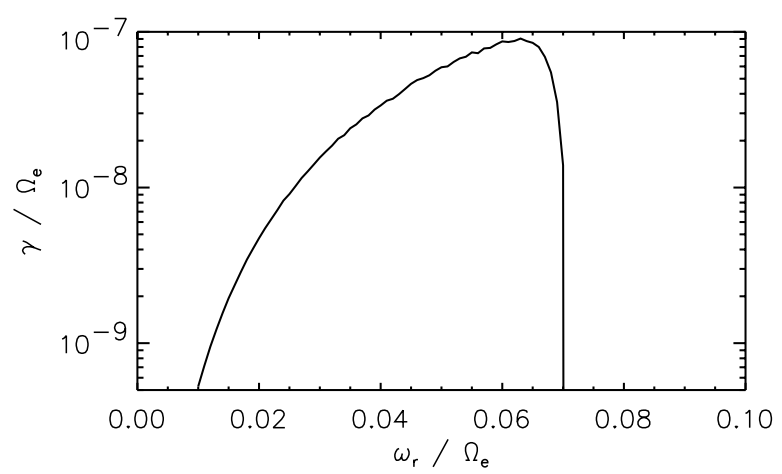

Fig. 5. Wave growth rate, $\gamma$, as a function of the wave frequency, $\omega_{r}$, for waves propagating parallel to the background magnetic field.

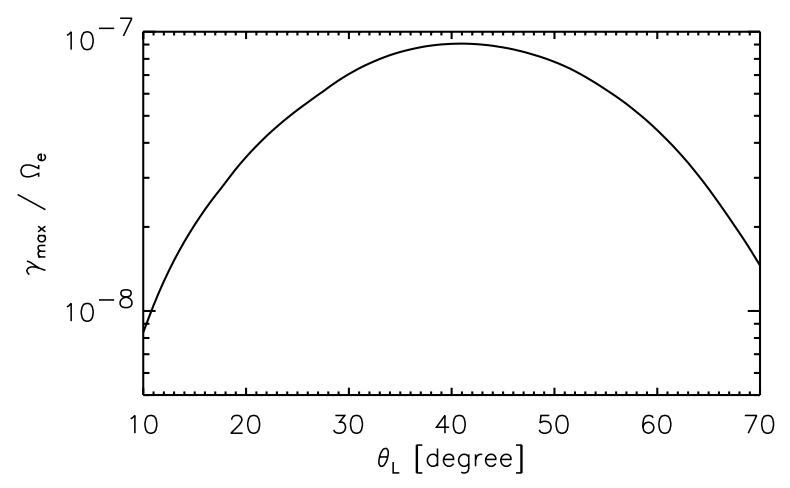

Fig. 6. Maximum wave growth rate, $\gamma_{\max }$, as a function of the loss-cone opening angle, $\theta_{\mathrm{L}}$.

parallel to the background magnetic field $(\theta=0)$ have the highest growth rates. In order to determine the maximum growth rate, $\gamma$ can be plotted as a function of the wave frequency, $\omega_{r}$, for a wave propagation angle $\theta=0$. Figure 5 shows the wave growth rate for $\theta=0$. For low frequencies, $\gamma$ increases with $\omega_{r}$. At $\omega_{r}=0.063 \Omega_{\mathrm{e}}$ it reaches a maximum and drops sharply at higher frequencies. This is due to the turn to negative $\gamma$ at $\omega_{r}=0.07 \Omega_{\mathrm{e}}$ that can be seen in Fig. 4 .

The reason for this behavior is the above mentioned dependence of the resonance frequency on the electron speed or momentum coordinate parallel to the background magnetic field. The higher the wave frequency, the lower the absolute value of $p_{\|}\left(p_{\|}<0\right)$. Thus, the integration path in the formula for the "imaginary" part of the dielectric tensor, Eq. (13), traverses regions with higher phase-space density as $\omega_{r}$ increases. Within the loss cone, this leads to an increase in the imaginary part of the dispersion function, and by means of Eq. (3) the wave growth rate increases. The maximum of $\gamma$ is reached when the resonance velocity enters the thermal core where there is no more loss cone VDF. For our model VDF (Fig. 3), this is the case for $p_{\|, \text {res }}=0.1 m_{\mathrm{e}} c$. For higher frequencies, the waves interact with the thermal core and are damped, as described in the previous subsection.

Since the loss cone provides the free energy for whistlerwave growth, its opening angle is a critical parameter. In a coronal loop, the angle depends on the position along the loop and the magnetic-field structure of the loop. The results that have been presented so far have been calculated for an opening angle of $\theta_{\mathrm{L}}=40^{\circ}$. Figure 6 shows the maximum wave growth rate as a function of the loss-cone opening angle. It is no surprise that the wave growth vanishes if the loss cone disappears, $\theta_{\mathrm{L}} \rightarrow 0$. With growing $\theta_{\mathrm{L}}$, the maximum wave growth rate increases and 
reaches a maximum for $\theta_{\mathrm{L}}=41^{\circ}$. For even higher $\theta_{\mathrm{L}}$, the maximum $\gamma$ decreases. For very wide loss cones, there is a low phasespace density in a wide area of the momentum space, $p_{\|}<0$, and Eq. (13) yields a smaller $\operatorname{Im}(\epsilon)$ and thus $\gamma$.

The results presented in this section show that whistler-wave generation by loss-cone distributions in flaring loops is well possible. The waves are generated with frequencies of less than one tenth of the electron cyclotron frequency. The corresponding wave velocities are in agreement with the observed intermediate frequency drift rates of fiber bursts (Kuijpers 1975; Benz \& Mann 1998). Thus, whistler waves originating from electron loss cone VDFs can lead to the emission of fiber bursts.

Figure 6 also allows for a determination of the maximum wave growth rate, which is possible in our model plasma. This maximum value is $\gamma_{\max }=9.1 \times 10^{-8} \Omega_{\mathrm{e}}=4.8 \mathrm{~s}^{-1}$. This corresponds to a wave-growth timescale of a few tenths of a second. It is noteworthy that this result is based on the model assumption that the hot electron component has a number density of $N_{\mathrm{h}}=10^{-6} N_{\mathrm{b}}$, with $N_{\mathrm{b}}$ the density of the thermal-background plasma. From the structure of the "imaginary" part of the dielectric tensor, Eq. (13), it follows that $\operatorname{Im}(\epsilon)$ is proportional to $N_{\mathrm{h}}$. Since it was found at the end of Sect. 2 that the imaginary part of the dispersion function, $D_{i}$, is of first order in $\operatorname{Im}(\epsilon)$, $D_{\mathrm{i}}$ and thus $\gamma$ scale linearly with $N_{\mathrm{h}}$. Thus, the assumption of e.g. $N_{\mathrm{h}}=10^{-5} N_{\mathrm{b}}$ would result in a maximum wave growth rate of $48 \mathrm{~s}^{-1}$.

\subsection{Magnetic field dependence}

The results in the previous section have been calculated for a background magnetic field of $B=3 \mathrm{G}$ that is typical of the height within a coronal loop where the electron background density is $N_{\mathrm{b}}=1.33 \times 10^{15} \mathrm{~m}^{-3}$, which corresponds to a plasma frequency of $f_{\mathrm{p}}=327 \mathrm{MHz}$ where fiber bursts are usually observed (Aurass et al. 2005). This magnetic field and plasma density lead to a ratio of $\omega_{\mathrm{p}} / \Omega_{\mathrm{e}}=39$ that is normal for the solar corona (Vocks \& Mann 2004).

The choice of $B$ with $\Omega_{\mathrm{e}}$ determines an important plasma parameter. Thus it is worthwhile to study the effect of this choice on the efficiency of the whistler-wave generation. Since the frequency of fiber bursts corresponds to the local plasma frequency at their source region (Kuijpers 1975), $\omega_{\mathrm{p}} / \Omega_{\mathrm{e}}>1$ is a necessary condition for their generation and subsequent observation on Earth, which imposes an upper limit on the magnetic field. On the other hand, the stability of the magnetic loop requires that the ratio between the plasma's thermal gas pressure and the energy density of the magnetic field, i.e. the plasma beta, is less than unity, $\beta<1$. This leads to a lower limit for $B$. In our model plasma, $\beta \approx 1$ for $B=3 \mathrm{G}$, so only stronger $B$ and thus lower ratios $\omega_{\mathrm{p}} / \Omega_{\mathrm{e}}$ are studied here. Since it has been found in Sect. 3.2 that the whistler-wave growth is strongest for waves propagating parallel to the background magnetic field, we concentrate on this propagation direction.

A series of calculations is performed with magnetic fields of $B=4,6,12,29$, and $58 \mathrm{G}$, that correspond to ratios $\omega_{\mathrm{p}} / \Omega_{\mathrm{e}}=30$, 20, 10, 4, and 2; see also Table 1 below. The electron VDF is in all cases the same as the one discussed in Sect. 3.1 with a thermal background component and a hot, nonthermal component with a loss cone that has an opening angle of $\theta_{\mathrm{L}}=40^{\circ}$. The VDF has been plotted in Fig. 3 .

Figure 7 displays the growth rates of whistler waves for the different magnetic fields as functions of the wave frequency. It is evident that the wave frequencies shift towards the electron cyclotron frequency, $\Omega_{\mathrm{e}}$, with increasing $B$. This is a consequence
Table 1. Maximum wave growth rates, $\gamma_{\max }$, for different $B$.

\begin{tabular}{ccc}
\hline \hline$B(\mathrm{G})$ & $\omega_{\mathrm{p}} / \Omega_{\mathrm{e}}$ & $\gamma_{\max }\left(\mathrm{s}^{-1}\right)$ \\
\hline 4 & 30 & 4.2 \\
6 & 20 & 4.8 \\
12 & 10 & 4.5 \\
29 & 4 & 2.8 \\
58 & 2 & 1.2 \\
\hline
\end{tabular}

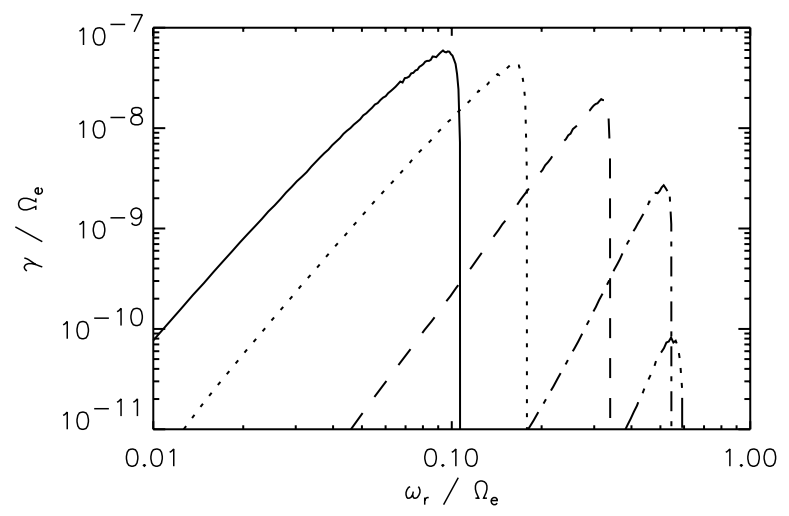

Fig. 7. Wave growth rate, $\gamma$, as a function of the wave frequency, $\omega_{r}$, for a loss-cone opening angle $\theta_{\mathrm{L}}=40^{\circ}$. The background magnetic field is $B=4 \mathrm{G}$ (solid line), $B=6 \mathrm{G}$ (dotted line), $B=12 \mathrm{G}$ (dashed line), $B=29 \mathrm{G}$ (dash-dotted line), and $B=58 \mathrm{G}$ (dash-dot-dotted line).

of the whistler-wave phase speed increasing with $B$. It was noted in the previous section that the maximum wave growth rate is reached for waves that are in resonance with electrons with a momentum of $p_{\|}=0.1 m_{\mathrm{e}} c$. For a given $p_{\|}$, the Doppler shift of the resonance frequency, $\omega_{\text {res }}$, decreases with increasing wave phase speed, and thus $\omega_{\text {res }}$ approaches $\Omega_{\mathrm{e}}$ as $B$ increases.

From Fig. 7 it also can be seen that the maximum values of $\gamma / \Omega_{\mathrm{e}}$ only slightly decrease with $B$ for the first three values of $B$ where the limit $\omega_{\mathrm{p}} / \Omega_{\mathrm{e}} \gg 1$ is still a valid approximation. But for $B=29 \mathrm{G}$ and even more for $B=58 \mathrm{G}$, i.e. $\omega_{\mathrm{p}} / \Omega_{\mathrm{e}}=2$ and thus close to unity, the growth rate drops sharply, so the whistler-wave generation mechanism seems to be less efficient for small $\omega_{\mathrm{p}} / \Omega_{\mathrm{e}}$.

The results shown in Fig. 7 were obtained for a loss-cone opening angle of $\theta_{\mathrm{L}}=40^{\circ}$. It is interesting to investigate the dependence of the maximum wave growth rate on $\theta_{\mathrm{L}}$ as $B$ is varied. Figure 8 shows these rates for the different magnetic fields as functions of the loss-cone opening angle. It is found again that for weak magnetic fields and thus high $\omega_{\mathrm{p}} / \Omega_{\mathrm{e}} \gg 1$ there is little variation with $B$. The overall maximum of $\gamma$ is reached for loss-cone opening angles in the range $\theta_{\mathrm{L}}=40-50^{\circ}$, and $\gamma_{\max } / \Omega_{\mathrm{e}}$ decreases slightly with $B$. But as soon as the limit $\omega_{\mathrm{p}} / \Omega_{\mathrm{e}} \gg 1$ is no longer valid, i.e. for $B=29 \mathrm{G}$ and higher, $\gamma_{\max }$ drops sharply. The decrease is stronger for small opening angles, $\theta_{\mathrm{L}}<40^{\circ}$, than for large angles, $\theta_{\mathrm{L}}>50^{\circ}$. As a consequence, the maximum value of $\gamma_{\max }$ shifts towards higher $\theta_{\mathrm{L}}$.

In Figs. 7 and 8 the maximum wave growth rate has been displayed in a normalized form, $\gamma_{\max } / \Omega_{\mathrm{e}}$. But since $\Omega_{\mathrm{e}}$ is proportional to $B$, it is illustrative to look at the maximum wave growth rate that is possible in the model plasma in absolute units. Table 1 shows the maximum values for $\gamma_{\max }$ for the different magnetic fields. For small $B$ and correspondingly high $\omega_{\mathrm{p}} / \Omega_{\mathrm{e}}$, $\gamma_{\max }$ has values around $4.5 \mathrm{~s}^{-1}$. These numbers agree with the $\gamma_{\max }=4.8 \mathrm{~s}^{-1}$ that has been found in the previous section for $B=3 \mathrm{G}$, so the increase in $\Omega_{\mathrm{e}}$ with increasing $B$ compensates 


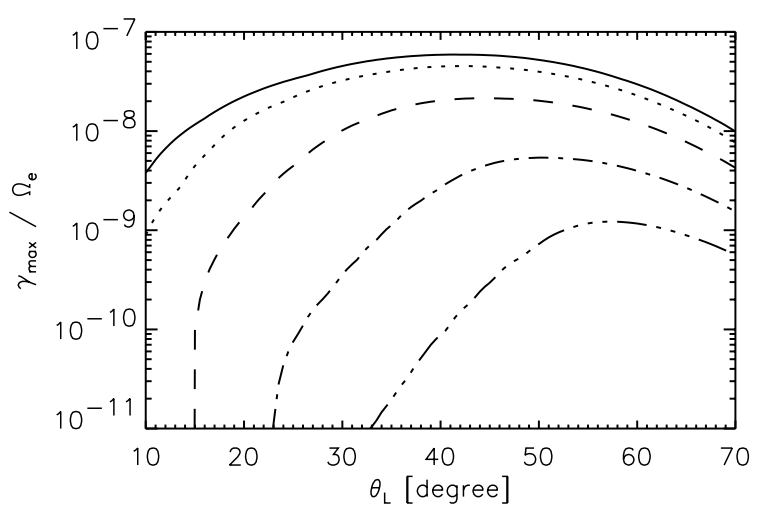

Fig. 8. Maximum wave growth rate, $\gamma_{\max }$, as a function of the loss-cone opening angle, $\theta_{\mathrm{L}}$, for background magnetic fields of $B=4 \mathrm{G}$ (solid line), $B=6 \mathrm{G}$ (dotted line), $B=12 \mathrm{G}$ (dashed line), $B=29 \mathrm{G}$ (dashdotted line), and $B=58 \mathrm{G}$ (dash-dot-dotted line).

the slight decrease of $\gamma_{\max } / \Omega_{\mathrm{e}}$ for small $B$ in Fig. 8. It can thus be concluded that the wave growth rates are fairly insensitive to the magnetic field of the loop. But for higher $B$ where $\omega_{\mathrm{p}} / \Omega_{\mathrm{e}}$ approaches unity, the maximum wave growth rate still drops with increasing $B$, but not as strong as indicated by Fig. 8, so the whistler-wave generation mechanism becomes less efficient for small $\omega_{\mathrm{p}} / \Omega_{\mathrm{e}}$.

\section{Conclusions and summary}

In this paper the generation of whistler waves by electron losscone distributions as they form in coronal loops during solar flares has been studied. The main objective was to investigate whether the resulting whistler-wave growth is fast enough to account for the generation of fiber bursts. The wave growth rates, $\gamma$, that are caused by a given electron VDF were determined as a function of the wave frequency, $\omega_{r}$, and propagation angle, $\theta$, towards the background magnetic field. Due to the Taylor expansion of the dispersion function, Eq. (3), this method is especially appropriate for low wave growth rates, $\gamma \ll \omega_{r}$.

Plotting the wave growth rate as a function of $\omega_{r}$ and $\theta$ clearly shows a region of wave growth. Investigation of the dependence of $\gamma$ on the propagation angle is especially interesting. It demonstrates the important role of higher order resonances even for very small $\theta$. An analytical calculation that considers only the case $\theta=0$ cannot reveal this restriction on the whistlerwave growth.

This result has some implications for the standard model of fiber-burst generation. If a whistler wave has been generated with a propagation angle $\theta=0$, where the wave growth is strongest, it propagates along the background magnetic field. But the loop is curved, so that a wave vector that is initially parallel to the magnetic field will deviate from $\boldsymbol{B}$ some time later. But if the index of refraction inside the loop decreases towards the lateral boundary of the loop (Kuijpers 1975; Roberts 2000), the wave is refracted back towards the magnetic-field lines. Thus, the whistler wave is forced along the loop, and the wave growth can continue for a sufficient time despite the strong limitation to small propagation angles.

Since whistler waves that propagate along the background magnetic field with $\theta=0$ have the highest growth rates, it is sufficient to study this case while looking for the maximum value of $\gamma$ in the model plasma. At low frequencies, $\gamma$ increases with increasing $\omega_{r}$ since the electron momentum needed to fulfill the resonance condition, Eq. (8), decreases. In momentum space, the resonance moves towards regions with higher electron phasespace density. But as soon as the resonance reaches the thermal core of the VDF, the growth rate reaches a maximum value $\gamma_{\max }$ and drops sharply at higher frequencies. This course defines the maximum wave growth rate for the given electron distribution.

The maximum value of $\gamma$ that is found in our model plasma varies with the opening angle of the loss cone. The absolute maximum is reached at $41^{\circ}$, with $\gamma_{\max }=9.1 \times 10^{-8} \Omega_{\mathrm{e}}=4.8 \mathrm{~s}^{-1}$, where $\gamma_{\max }$ has values of more than $4 \mathrm{~s}^{-1}$ over a wide range of loss cone opening angles from $32^{\circ}-50^{\circ}$. Since fiber bursts are believed to be generated by the interaction between whistlers and electrostatic modes, their pulse duration at a fixed frequency should not be less than the growth time of the whistler wave. Observations (Aurass et al. 2005) show pulse durations of some tenths of a second at $327 \mathrm{MHz}$. Thus, the values of $\gamma_{\max }$ that result from our model indicate a whistler-wave growth that is fast enough to account for the observed pulse durations. These values are based on the assumption that the hot, nonthermal component of the electron distribution has a low number density $N_{\mathrm{h}}=10^{-6} N_{\mathrm{b}}$, with $N_{\mathrm{b}}$ being the density of the background electrons. A higher $N_{\mathrm{h}}$ would lead to higher growth rates.

A study of the whistler wave generation for different magnetic fields shows that the maximum wave growth rate is fairly independent of $B$ as long as $B$ is small enough that $\omega_{\mathrm{p}} / \Omega_{\mathrm{e}} \gg 1$ is a valid assumption. The dependence of $\gamma_{\max }$ on the loss-cone opening angle also hardly depends on $B$ in this regime. But for higher $B$ with $\omega_{\mathrm{p}} / \Omega_{\mathrm{e}} \approx 1$, the growth rates decrease rapidly. However, this finding has no implications for the wave generation in the solar corona where condition $\omega_{\mathrm{p}} / \Omega_{\mathrm{e}} \gg 1$ is normally met (Vocks \& Mann 2004). Thus, the wave generation does not depend critically on the actual value of the background magnetic field, $B$. The whistler-wave growth is fast enough to account for the observed fiber bursts over a wide range of magnetic fields and loss-cone opening angles. This result corroborates the present model (Aurass et al. 2005) of fiber bursts as being caused by whistler-wave packets traveling along a magnetic loop.

Acknowledgements. The authors are grateful to A. Warmuth for providing the photon and electron data in Fig. 1, and to H. Aurass and G. Rausche for preparing the fiber-burst plot in Fig. 2.

\section{References}

Aurass, H., Rausche, G., Mann, G., \& Hofmann, A. 2005, A\&A, 435, 1137 Baumjohann, W., \& Treumann, R. A. 1996, Basic Space Plasma Physics (London: Imperial College Press)

Benz, A. O., \& Mann, G. 1998, A\&A, 333, 1034

Brown, J. C. 1971, Sol. Phys., 18, 489

Croley, D. R., Jr., Mizera, P. F., \& Fennell, J. F. 1978, J. Geophys. Res., 83, 2701

Helliwell, R. A. 1975, Phil. Trans. Roy. Soc. London A, 280, 137

Holman, G. D., Sui, L., Schwartz, R. A., \& Emslie, A. G. 2003, ApJ, 595, L97

Kuijpers, J. 1975, Sol. Phys., 44, 173

Lin, R. P. 1974, Space Sci. Rev., 16, 189

Lin, R. P., Dennis, B. R., Hurford, G. J., et al. 2002, Sol. Phys., 210, 3

Mann, G., Karlický, M., \& Motschmann, U. 1987, Sol. Phys., 110, 381

Mann, G., Baumgaertel, K., Chernov, G. P., \& Karlický, M. 1989, Sol. Phys., 120, 383

Mann, G., Aurass, H., Voigt, W., \& Paschke, J. 1992, Proc. First SOHO Workshop, ESA SP-348, 129

Matsuda, Y., \& Smith, G. R. 1992, J. Comp. Phys., 100, 229

Melrose, D. B. 1980, Plasma Astrophysics (New York: Gordon and Breach)

Melrose, D. B. 1986, Instabilities in Space and Laboratory Plasmas (Cambridge: Cambridge University Press)

Montgomery, D. C., \& Tidman, D. A. 1964, Plasma Kinetic Theory (New York: McGrawHill)

Roberts, B. 2000, Sol. Phys., 193, 139

Scharer, J. E., \& Trivelpiece, A. W. 1967, Phys. Fluids, 10, 591

Vocks, C., \& Mann, G. 2004, A\&A, 419, 763

Warmuth, A., \& Mann, G. 2004, in Space Weather, ed. K. Scherer, H. Fichtner, B. Heber, \& U. Mall, Lecture Notes in Physics, 656, 49 (Berlin: Springer)

Wu, C. S., \& Freund, H. P. 1977, ApJ, 213, 575

Wu, C. S., \& Lee, L. C. 1979, ApJ, 230, 621

Xiao, F., Thorne, R. M., Gurnett, D. A., \& Williams, D. J. 2003, GRL, 30, SSC 3-1 